\title{
Long-Term Outcomes of Local Excision Following Neoadjuvant Chemoradiotherapy for Locally Advanced Rectal Cancer
}

\author{
Lucrezia D'Alimonte, MD¹, Quoc Riccardo Bao, MD¹, Gaya Spolverato, MD', Giulia Capelli, MD', \\ Paola Del Bianco, $\mathrm{MS}^{2}$, Laura Albertoni, $\mathrm{MD}^{3}$, Antonino De Paoli, $\mathrm{MD}^{4}$, Mario Guerrieri, $\mathrm{MD}^{5}$, \\ Giovanna Mantello, $\mathrm{MD}^{6}$, Maria Antonietta Gambacorta, $\mathrm{MD}^{7}$, Vincenzo Canzonieri, $\mathrm{MD}^{8}$, \\ Vincenzo Valentini, $\mathrm{MD}^{7}$, Claudio Coco, $\mathrm{MD}^{9}$, and Salvatore Pucciarelli, $\mathrm{MD}^{1}$ \\ ${ }^{1}$ Department of Surgical, Oncological, and Gastroenterological Sciences, First Surgical Clinic, University of Padua, Padua, \\ Italy; ${ }^{2}$ Clinical Research Unit, Istituto Oncologico Veneto IOV - IRCCS, Padua, Italy; ${ }^{3}$ Surgical Pathology and \\ Cytopathology Unit, Department of Medicine-DIMED, University of Padua, Padua, Italy; ${ }^{4}$ Department of Radiation \\ Oncology, National Cancer Institute, Aviano, Italy; ${ }^{5}$ General Surgery, Marche Polytechnic University, Ancona, Italy; \\ ${ }^{6}$ Department of Radiotherapy, State Hospital, Ancona, Italy; ${ }^{7}$ Department of Radiotherapy, Catholic University of Rome, \\ Rome, Italy; ${ }^{8}$ Department of Pathology, National Cancer Institute, Aviano, Italy; ${ }^{9}$ Department of Surgical Sciences, \\ Catholic University of Rome, Rome, Italy
}

\begin{abstract}
Background. Local excision might represent an alternative to total mesorectal excision for patients with locally advanced rectal cancer who achieve a major or complete clinical response after neoadjuvant chemoradiotherapy.

Methods. Between August 2005 and July 2011, 63 patients with mid-low rectal adenocarcinoma who had a major/complete clinical response after neoadjuvant chemoradiotherapy were enrolled in a multicenter prospective phase 2 trial and underwent transanal full thickness local excision. The main endpoint of this study was to evaluate the 5- and 10-year overall, relapse-free, local, and distant relapse-free survival, which were calculated by applying the Kaplan-Meier method. The rate of patients with rectum preserved and without stoma were also calculated.
\end{abstract}

Results. Of 63 patients, 38 (60\%) were male and $25(40 \%)$ were female, with a median (range) age of 64 (25-82) years. At baseline, the following clinical stages were found: cT2, $n=21$ (33.3\%); cT3, $n=42$ (66.6\%), 39

(C) The Author(s) 2020

First Received: 2 July 2020

Accepted: 28 August 2020;

Published Online: 30 October 2020

G. Spolverato, MD

e-mail: gaya.spolverato@unipd.it
(61.9\%) patients were $\mathrm{cN}+$. At a median (range) follow-up of 108 (32-166) months, the estimated cumulative 5- and 10-year overall survival, relapse-free survival, local recurrence-free survival, and distant recurrence-free survival were $87 \%$ (95\% CI 76-93) and 79\% (95\% CI 66-87), $89 \%$ (95\% CI 78-94) and $82 \%$ (95\% CI 66-91), both $91 \%$ (95\% CI 81-96), and 90\% (95\% CI 80-95) and 86\% (95\% CI 73-93), respectively. Overall, 49 (77.8\%) patients had their rectum preserved, and $54(84.1 \%)$ were stoma-free.

Conclusion. In highly selected patients, the local excision approach after neoadjuvant chemoradiotherapy is associated with excellent long-term outcomes, high rates of rectum preservation and absence of permanent stoma.

The standard of care for locally advanced rectal cancer is neoadjuvant chemoradiotherapy followed by total mesorectal excision (TME). This strategy has been shown to decrease the rate of local recurrence up to $6 \%^{1}$ with estimated 5- and 10-year overall survival (OS) of approximately 75 and $60 \%,{ }^{1,2}$ respectively. Moreover, after neoadjuvant chemoradiotherapy, up to $28 \%$ of patients show a pathological complete response (pCR), ${ }^{3}$ and a further $20 \%$ show a major (few residual cancer cells) pathological response. ${ }^{4}$ These findings are clinically relevant as patients with pCR to neoadjuvant chemoradiotherapy followed by TME show a significantly better outcome compared with non-responders. ${ }^{5}$ However, 
TME is associated with higher rates of morbidity, impairment of bowel function and quality of life ${ }^{6}$ and permanent stoma.

The above considerations explain the increasing interest in rectum-sparing approaches, either local excision (LE) or watch-and-wait, for patients with complete or major clinical response to neoadjuvant chemoradiotherapy. The transanal LE approach has been used for many years in patients unfit or refusing major surgery and has been evaluated by several retrospective studies. More recently, a few prospective trials have been performed in the setting of a rectum-sparing strategy for patients with both major and complete clinical response. ${ }^{7-11}$ These studies found that patients who underwent LE had comparable short-term oncological outcomes, associated with better bowel function and quality of life, ${ }^{12}$ and reduced rates of complications compared with those who underwent standard TME. ${ }^{10,13}$ While these studies report on short-term outcomes, there is a lack of information related to the longterm outcomes. ${ }^{14,15}$

The present study aimed to evaluate long-term survival outcomes of rectal cancer patients who underwent LE following neoadjuvant chemoradiotherapy.

\section{METHODS}

\section{Study Design}

This study is an update of a previously published prospective multicenter phase 2 trial that enrolled patients who underwent LE after neoadjuvant chemoradiotherapy in four Italian centers. ${ }^{8}$ The trial was approved by the local ethics committee of each center involved in the study. Inclusion criteria, and patients, tumor, and treatment characteristics have been previously reported. ${ }^{8}$ The inclusion criteria were: age $>18$ years, histologically confirmed rectal adenocarcinoma, located up to $11 \mathrm{~cm}$ from anal verge. Either clinical T3 or low-lying T2 tumors, with a major (complete or near complete) clinical response to neoadjuvant chemoradiotherapy were included. The baseline work-up included clinical history, digital rectal examination, colonoscopy, carcino-embryonic antigen level, chest/abdomen computed tomography scan and pelvic magnetic resonance imaging (MRI). Major clinical response has been previously defined as the absence of a positive regional lymph node on MRI, and either no mucosal abnormality or a flat residual scar (complete response), or a superficial ulcer less than $2 \mathrm{~cm}$ at proctoscopy (major response). ${ }^{16}$ The differentiation between complete and major clinical response was not formally required when the protocol was planned. Clinical and pathological TNM staging were reported according to the
American Joint Committee on Cancer, 8th Edition. ${ }^{17}$ The following histopathological data were collected: yT stage, tumor regression grade (TRG) ${ }^{18}$ status of resection margin, tumor differentiation, and vascular and lymphatic invasion. The pathologic response was defined as complete (pCR) if no viable tumor cells were found in the surgical specimen. When residual cancer was found, the pathologist was requested to report on the presence of the following unfavorable features: $\mathrm{pT}>1$, lympho-vascular invasion, poor differentiation grade, involved margin, and TRG $>2$.

\section{Treatment Details}

Fluoropyrimidine-based chemotherapy was administered concomitantly with radiotherapy at a total dose of $50.4 \mathrm{~Gy}$, given in 28 fractions of 1.8 Gy each. Patients were re-staged at least 5 weeks after the completion of neoadjuvant chemoradiotherapy and those with major response were considered eligible for $\mathrm{LE}$ and enrolled in the study. After signing the informed consent, the patients underwent a full-thickness excision using the traditional transanal approach or transanal endoscopic microsurgery (TEM).

While patients with pCR (ypT0), or with ypT1 and histopathologically favorable features were observed, those with residual cancer showing at least one of the unfavorable histopathological features were recommended to undergo a subsequent completion TME surgery.

\section{Long-Term Outcomes Definition and Statistical Analysis}

Patients underwent a strict follow-up, the modalities of which have been detailed elsewhere. ${ }^{8}$ Local recurrence was defined as any pelvic endoluminal or extraluminal recurrence, while recurrences outside the pelvis were defined as distant. To evaluate the overall survival (OS), relapse-free survival (RFS), local relapse-free survival (LRFS), and distant relapse-free survival (DRFS), the Kaplan-Meier method was used. Each outcome was calculated from the date of LE to the date of the event (local, and distant recurrence, death, or the last follow-up). The proportion of patients without stoma or with rectum preserved were also calculated. All analyses were carried out with STATA version 13.0 (StataCorp, College Station, TX).

\section{RESULTS}

\section{Patient, Tumor, and Treatment Characteristics}

Between August 2005 and July 2011, 63 patients were enrolled in the study. The baseline characteristics of 
patients, tumor, and treatment modality have been previously reported ${ }^{8}$ and are summarized in Table 1.

\section{Histopathology}

The histopathological tumor characteristics and post-LE treatment details are summarized in Table 2. Interestingly, out of 42 patients who showed a pCR, only 19 (45.2\%) were clinically considered to be complete responders (ycT0), the remaining $23(54.8 \%)$ had a major clinical response which was considered near complete. Therefore, on the basis of favorable histopathological features in one patient, a total of 43 patients were observed. A completion radical surgery was recommended in the remaining 20 patients. Of them, 11 underwent a TME (low anterior resection, $n=7$; abdominoperineal resection, $n=4$ ), two underwent a transanal local re-excision, and seven refused any further surgery (Fig. 1).

\section{Outcomes}

Long-term survival outcomes are summarized in Fig. 2. At a median (range) follow-up of 108 (32-166) months, the estimated cumulative 5- and 10-year OS were $87 \%(95 \%$ CI 76-93) and 79\% (95\% CI 66-87), respectively (Fig. 2a). The estimated cumulative 5- and 10-year RFS were $89 \%$ (95\% CI 78-94) and $82 \%$ (95\% CI 66-91), respectively (Fig. 2b).

The estimated cumulative 5- and 10-year LRFS were both $91 \%$ (95\% CI 81-96) (Fig. 2c). Overall, five (8\%) patients were found to have a local recurrence, which occurred within the first 5 years of follow-up. Two recurrences were endoluminal and three extra-luminal. Characteristics of patients with local and distant recurrence are summarized in Table 3. Of five patients experiencing local recurrence, one refused further treatments, one underwent chemoradiotherapy for concomitant distant disease, and three underwent surgery (local re-excision, abdominoperineal resection, and low anterior resection + intraoperative radiotherapy). Of these five patients, four also showed distant recurrence and four died.

The estimated cumulative 5- and 10-year DRFS were 90\% (95\% CI 80-95), and 86\% (95\% CI 73-93), respectively (Fig. 2d). Eight (13\%) patients had distant recurrences. Of eight patients experiencing distant recurrences, five died.
TABLE 1 Patient, tumor, and treatment characteristics

\begin{tabular}{|c|c|c|}
\hline Variables & $N=63$ & $\%$ \\
\hline Age & Median (range), years & $64(25-82)$ \\
\hline \multicolumn{3}{|l|}{ Gender } \\
\hline Males & 38 & 60.3 \\
\hline Females & 25 & 39.7 \\
\hline \multicolumn{3}{|l|}{ Carcinoembryonic antigen level } \\
\hline$\leq 5 \mathrm{ng} / \mathrm{ml}$ & 37 & 58.7 \\
\hline$>5 \mathrm{ng} / \mathrm{ml}$ & 7 & 11.1 \\
\hline Missing & 19 & 30.2 \\
\hline \multicolumn{3}{|l|}{ Baseline clinical T stage } \\
\hline $\mathrm{cT} 2$ & 21 & 33.3 \\
\hline cT3 & 42 & 66.6 \\
\hline \multicolumn{3}{|l|}{ Baseline clinical $N$ stage } \\
\hline Negative & 24 & 38.1 \\
\hline Positive & 39 & 61.9 \\
\hline Tumor distance from anal verge & Median (range), $\mathrm{cm}$ & $5.8(3-11)$ \\
\hline \multicolumn{3}{|l|}{ Radiotherapy, total dose } \\
\hline$<50.4 \mathrm{~Gy}$ & 2 & 3.2 \\
\hline$>50.4$ Gy & 61 & 96.8 \\
\hline \multicolumn{3}{|l|}{ Chemotherapy } \\
\hline Capecitabine or 5-fluorouracil alone & 37 & 58.7 \\
\hline Capecitabine or 5-fluorouracil, and oxaliplatin & 24 & 38.1 \\
\hline Others & 2 & 3.2 \\
\hline \multicolumn{3}{|l|}{ Local excision technique } \\
\hline Transanal excision & 30 & 47.6 \\
\hline Transanal endoscopic microsurgery & 33 & 52.4 \\
\hline
\end{tabular}


TABLE 2 Histopathology characteristics of tumors after local excision, and subsequent surgical treatment of patients with unfavorable histopathology features

\begin{tabular}{|c|c|c|}
\hline Variables & $N=63$ & $\%$ \\
\hline \multicolumn{3}{|l|}{ ypT stage } \\
\hline T0 & 42 & 66.7 \\
\hline $\mathrm{T} 1$ & 4 & 6.3 \\
\hline $\mathrm{T} 2$ & 15 & 23.8 \\
\hline $\mathrm{T} 3$ & 2 & 3.2 \\
\hline \multicolumn{3}{|l|}{ Tumor regression grade } \\
\hline 1 & 42 & 66.7 \\
\hline 2 & 5 & 7.9 \\
\hline $3-5$ & 16 & 25.4 \\
\hline \multicolumn{3}{|l|}{ Margin } \\
\hline Negative & 59 & 93.7 \\
\hline Positive & 4 & 6.3 \\
\hline \multicolumn{3}{|c|}{ Subsequent completion radical surgery } \\
\hline Low anterior resection & 7 & 11.1 \\
\hline Abdominoperineal resection & 4 & 6.3 \\
\hline Local re-excision $^{\S}$ & 2 & 3.2 \\
\hline Refused & 7 & 11.1 \\
\hline Not required & 43 & 68.3 \\
\hline
\end{tabular}

${ }^{\S}$ Patients refused major surgery and only accepted minor (local excision) surgery histopathologically unfavorable features $(n=5)$, and salvage radical surgery for local recurrence $(n=2)$.

At the last follow-up, 13 patients $(20.6 \%)$ had died.

\section{DISCUSSION}

The aim of this study was to evaluate the long-term oncological outcomes of patients with rectal cancer who showed a major (complete or near complete) clinical response to neoadjuvant chemoradiotherapy and then underwent full thickness LE. The main findings of the study were that both OS and RFS were close to $90 \%$ at 5 years and close to $80 \%$ at 10 years; and that $78 \%$ of patients had the rectum preserved, and $84 \%$ were stomafree. These findings are encouraging and seem to support the hypothesis that LE may represent a safe alternative to TME, offering comparable results, minimal morbidity and better functional outcomes. ${ }^{12,14,15,19}$

Although with shorter follow-up, several prospective studies have been published in recent years. The American ACOSOG Z6041 multicenter phase 2 trial recruited 79 patients who underwent LE after neoadjuvant chemoradiotherapy. ${ }^{11}$ At a median follow-up of 56 months, the estimated 5-year OS and DFS were $90.9 \%$ and $79.3 \%$, respectively. As in our series, two out of five local recurrences were found in patients with a pCR. This is not

\begin{tabular}{|l|l|l|}
\hline Pathological response & Completion radical surgery & Long-term outcomes \\
\hline
\end{tabular}

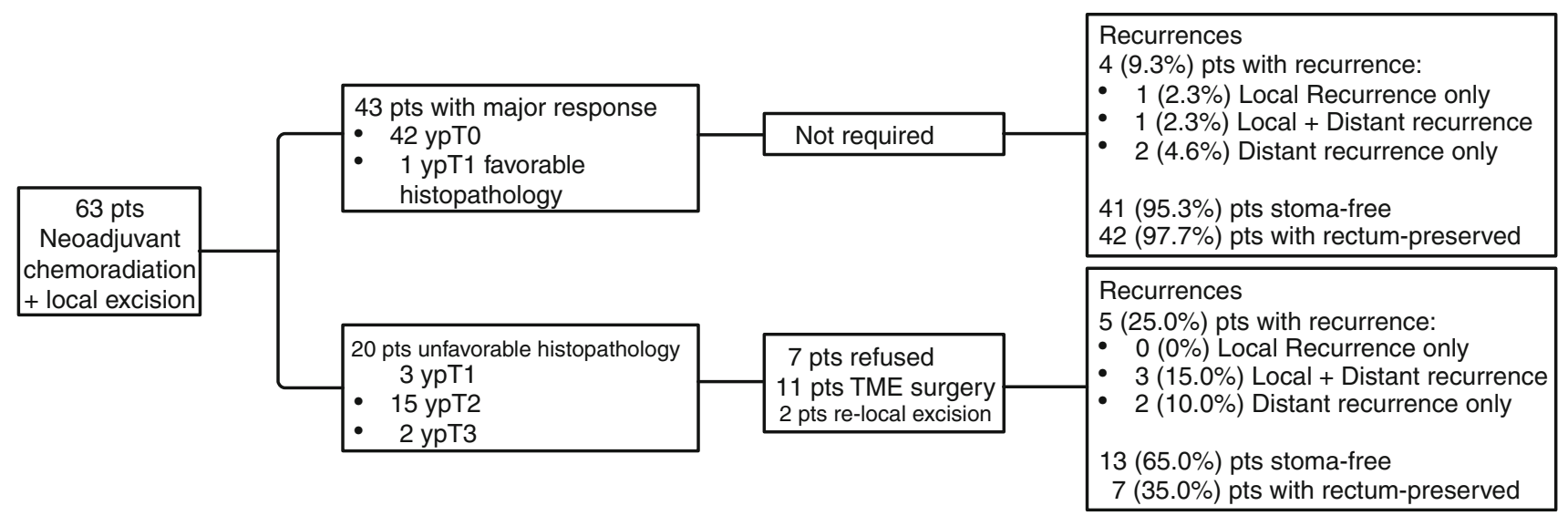

FIG. 1 Pathological response, completion radical surgery and long-term outcomes of the study group after a median follow-up of 108 months

Overall, $49(77.8 \%)$ patients had their rectum preserved, and $54(84.1 \%)$ were stoma-free. The reasons for a definitive stoma were fecal incontinence after sphincter saving surgery $(n=1)$, rectal stricture after LE $(n=1)$, completion surgery after LE because of the presence of surprising as the LE neither includes all the area of the pretreatment primary tumor, nor removes the mesorectal nodes. Moreover, an incomplete histopathological examination cannot be excluded. In their prospective trial, Lezoche et al. randomized 100 patients who, after 

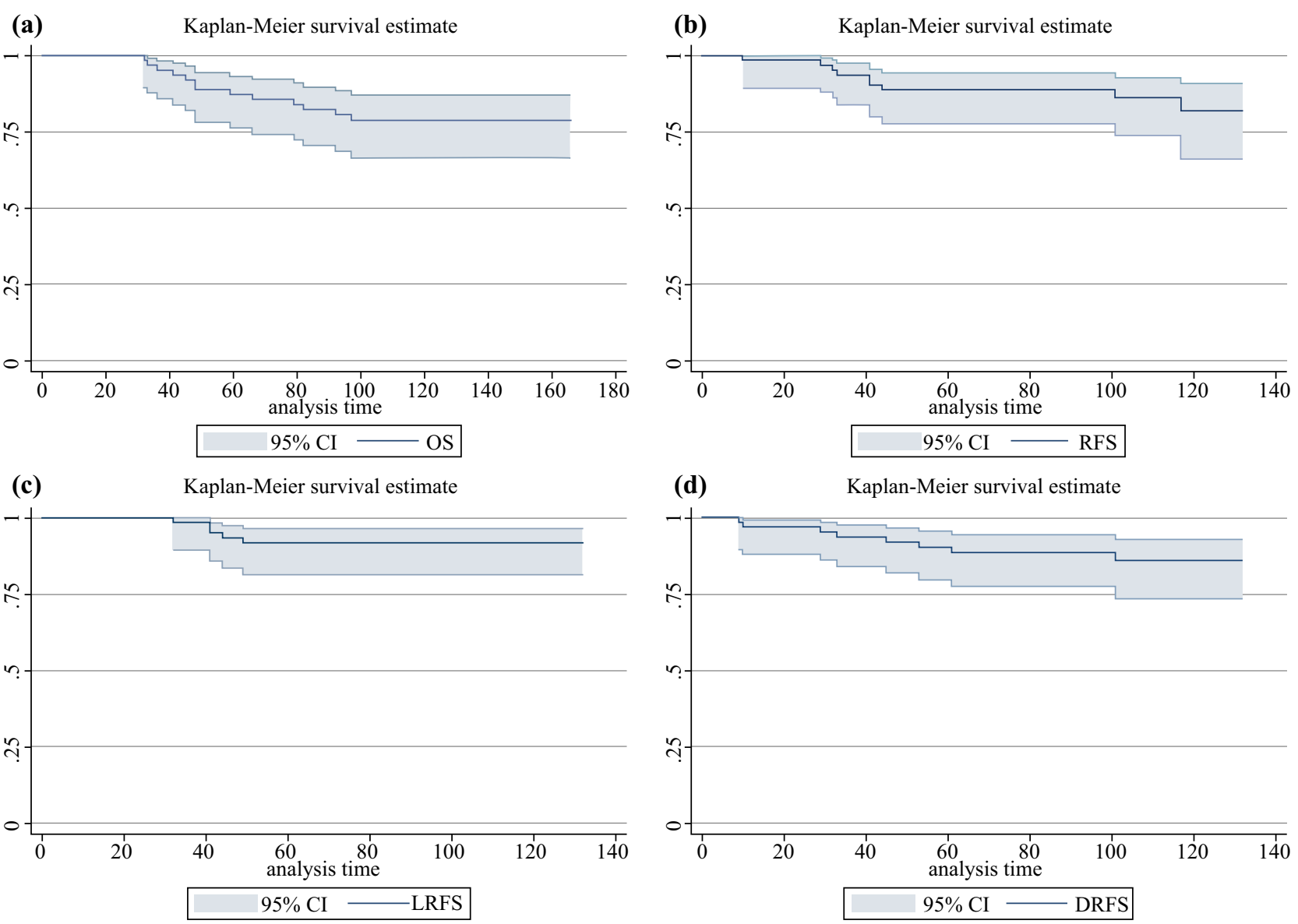

FIG. 2 Kaplan-Meier estimate. a Overall survival. b Relapse-free survival. c Local relapse-free survival. d Distant relapse-free survival

neoadjuvant chemoradiotherapy, underwent LE $(n=50)$ or standard laparoscopic TME $(n=50) .{ }^{19}$ At a median follow-up of 9.6 years, the cancer-related survival rate was $89 \%$ and the OS was $72 \%$, without any differences compared with the laparoscopic TME arm. The rate of local recurrence was $8 \%$. Although both previous trials included patients with favorable cases (small rectal cancer, clinically staged as cT2N0), the outcomes are comparable with our study. More recently, prospective studies included clinical T2-T3 rectal cancer. ${ }^{14,15}$ At a median follow-up of 60 months, Rullier et al. reported no difference between LE and TME arms, either in terms of 5-year local recurrence $(7 \%$ vs. $7 \%)$, or in terms of metastatic disease $(18 \%$ vs. $19 \%)$, OS ( $84 \%$ vs. $82 \%)$, or DFS $(70 \%$ vs. $72 \%) .{ }^{14}$ Furthermore, at a median follow-up of 53 months, Stijns et al. reported a 5-year actuarial local recurrence rate of $7.7 \%$, DFS of $81.6 \%$, and OS of $82.8 \%$, respectively. ${ }^{15}$ It should be noted that in our study, $60 \%$ of patients were staged as cT3 at baseline, whereas in the trials of Rullier et al. and Stijns et al. the rates of cT3 were $45 \%$ and $29 \%$, respectively. ${ }^{13,14}$ These findings may suggest that the rectum preservation strategy should be based on clinical response to neoadjuvant therapy instead of clinical baseline staging. A clear message derived from our and the previous trials is that the risk of local recurrence after LE is higher than after TME surgery. In order to reduce this risk, patients with unfavorable histologic features, particularly ypT2-3 tumors, should undergo an early completion radical surgery. In three of five patients with local recurrences, the completion radical surgery was refused. Patients should be informed that LE is basically an excisional biopsy and that there is an increased risk of local recurrence, particularly for those patients refusing the recommended completion radical surgery (Table 3). Moreover, as all local recurrences were observed between 31 and 49 months after LE, a close and prolonged follow-up should be strongly recommended. This close follow-up is also required in patients with a pCR, as local recurrences have been observed in these patients.

Although this study reports on the LE approach, some considerations related to the watch-and-wait approach seem appropriate. Compared with the watch-and-wait policy, the LE approach is associated with postoperative morbidity and the need for completion TME, which is recommended in up to one third of cases, ${ }^{8,14,15}$ and may be challenging. On the other hand, LE provides a histological 


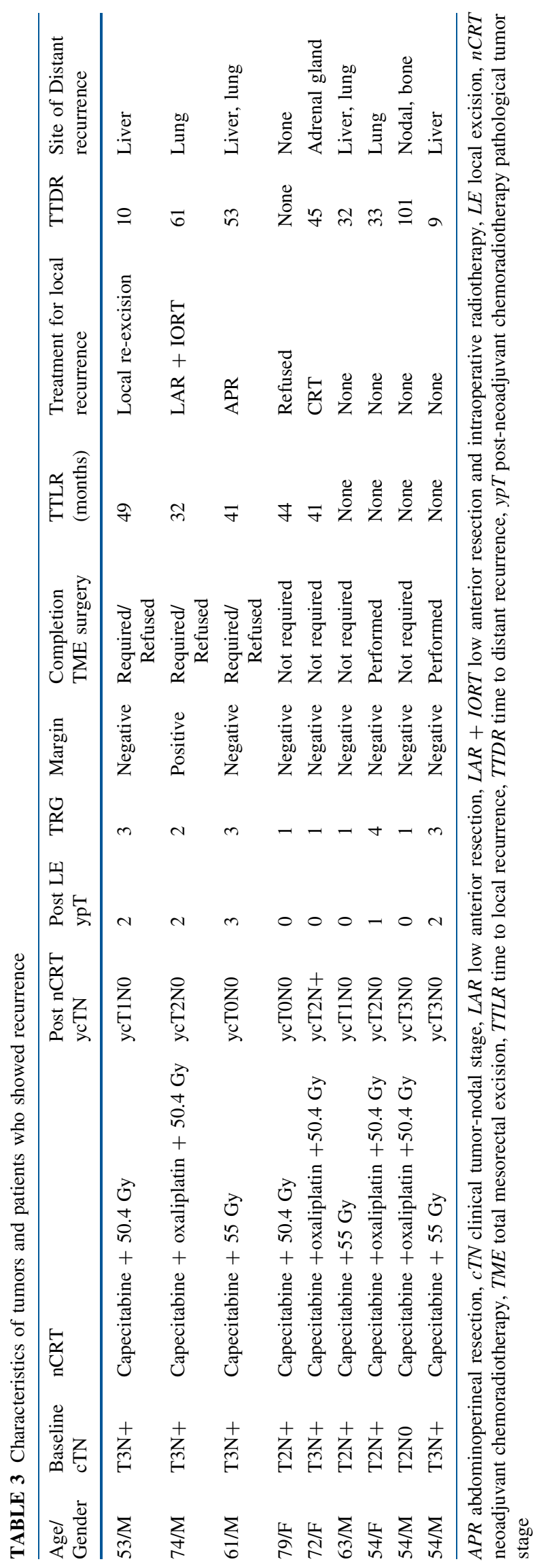


proof of pCR, avoiding the delayed diagnosis of regrowth; in these patients long-term impact on survival is matter or debate. ${ }^{20}$ In addition, while the watch-and-wait approach is only indicated in patients with clinical complete response, LE also seems appropriate in patients with a near complete clinical response. In the present study only 19 of 42 patients with a pCR were considered complete responders at restaging. Following the current indication (watch-andwait to be performed only in patients with clinical complete response) 23 of 42 patients with pCR would have undergone TME instead of rectum preservation. An alternative approach could be to use both strategies within a rectum sparing program: watch-and-wait in patients with clinical complete response and LE in those with a nearcomplete clinical response. Independently from the strategy used, the key point still relies on the improvement of patient selection by better staging accuracy. As depicted in Fig. 1, in the group of patients with a pathologic major response (ypT0 and ypT1 with favorable histology) the rates of local recurrence were less than 5\%, and more than $95 \%$ of patients were stoma-free with the rectum preserved.

The limitations of this study are related to the small sample size and to the lack of a comparative arm. Our institution is currently involved in a multicenter observational study (RESARCH), whose primary endpoint is to validate the rectal-sparing policy in patients with complete or near-complete clinical response after neoadjuvant chemoradiotherapy. ${ }^{16}$ Randomized trials in this field are challenging mainly due to the difficult accrual of patients. Likely, the best evidence may therefore derive from large observational prospective trials or national and international registry. ${ }^{21}$

\section{CONCLUSION}

The present study confirms that, in patients showing major clinical response after neoadjuvant chemoradiotherapy, the LE approach may offer, in the long-term, similar encouraging survival outcomes to those previously reported in studies with shorter follow-up. ${ }^{14,15,19,22}$ Moreover, about $80 \%$ of patients could have their rectum definitively preserved.

FUNDING SOURCE Open access funding provided by Università degli Studi di Padova within the CRUI-CARE Agreement. There was no external funding for this manuscript.

DISCLOSURES All the authors have indicated that they have no conflicts of interest to declare.

OPEN ACCESS This article is licensed under a Creative Commons Attribution 4.0 International License, which permits use, sharing, adaptation, distribution and reproduction in any medium or format, as long as you give appropriate credit to the original author(s) and the source, provide a link to the Creative Commons licence, and indicate if changes were made. The images or other third party material in this article are included in the article's Creative Commons licence, unless indicated otherwise in a credit line to the material. If material is not included in the article's Creative Commons licence and your intended use is not permitted by statutory regulation or exceeds the permitted use, you will need to obtain permission directly from the copyright holder. To view a copy of this licence, visit http://creativecommons. org/licenses/by/4.0/.

\section{REFERENCES}

1. Sauer R, Becker H, Hohenberger W, et al. Preoperative versus postoperative chemoradiotherapy for rectal cancer. $N$ Engl J Med. 2004;351(17):1731-40.

2. Sauer R, Liersch T, Merkel S, et al. Preoperative versus postoperative chemoradiotherapy for locally advanced rectal cancer: results of the German CAO/ARO/AIO-94 randomized phase III trial after a median follow-up of 11 years. J Clin Oncol. 2012;30(16):1926-33.

3. Valentini V, Gambacorta MA, Cellini F, et al. The INTERACT Trial: Long-term results of a randomised trial on preoperative capecitabine-based radiochemotherapy intensified by concomitant boost or oxaliplatin, for cT2 (distal)-cT3 rectal cancer. Radiother Oncol. 2019;134:110-8.

4. Gérard J-P, Azria D, Gourgou-Bourgade S, et al. Comparison of two neoadjuvant chemoradiotherapy regimens for locally advanced rectal cancer: results of the phase III trial ACCORD 12/0405-Prodige 2. J Clin Oncol. 2010;28(10):1638-44.

5. Maas M, Nelemans PJ, Valentini V, et al. Long-term outcome in patients with a pathological complete response after chemoradiation for rectal cancer: a pooled analysis of individual patient data. Lancet Oncol. 2010;11(9):835-44.

6. Bushati M, Pucciarelli S, Gennaro N, et al. Local excision in rectal cancer patients with major or complete clinical response after neoadjuvant therapy: a case-matched study. Int J Colorectal Dis. 2019;34(12):2129-36.

7. Bujko K, Richter P, Smith FM, et al. Preoperative radiotherapy and local excision of rectal cancer with immediate radical reoperation for poor responders: A prospective multicentre study. Radiother Oncol. 2013;106(2):198-205.

8. Pucciarelli S, De Paoli A, Guerrieri M, et al. Local excision after preoperative chemoradiotherapy for rectal cancer: results of a multicenter phase II clinical trial. Dis Colon Rectum. 2013;56(12):1349-56.

9. Verseveld M, de Graaf EJ, Verhoef C, et al. Chemoradiation therapy for rectal cancer in the distal rectum followed by organsparing transanal endoscopic microsurgery (CARTS study). Br J Surg. 2015;102(7):853-60.

10. Rullier E, Rouanet P, Tuech JJ, et al. Organ preservation for rectal cancer (GRECCAR 2): a prospective, randomised, openlabel, multicentre, phase 3 trial. Lancet. 2017;390(10093):469-79.

11. Garcia-Aguilar J, Renfro LA, Chow OS, et al. Organ preservation for clinical T2NO distal rectal cancer using neoadjuvant chemoradiotherapy and local excision (ACOSOG Z6041): results of an open-label, single-arm, multi-institutional, phase 2 trial. Lancet Oncol. 2015;16(15):1537-46.

12. Pucciarelli S, Giandomenico F, De Paoli A, et al. Bowel function and quality of life after local excision or total mesorectal excision following chemoradiotherapy for rectal cancer. $\mathrm{Br} J$ Surg. 2017;104(1):138-47. 
13. Restivo A, Zorcolo L, D'Alia G, et al. Risk of complications and long-term functional alterations after local excision of rectal tumors with transanal endoscopic microsurgery (TEM). Int $J$ Colorectal Dis. 2016;31(2):257-66.

14. Rullier E, Vendrely V, Asselineau J, et al. Organ preservation with chemoradiotherapy plus local excision for rectal cancer: 5-year results of the GRECCAR 2 randomised trial. Lancet Gastroenterol Hepatol. 2020;5(5):465-74.

15. Stijns RCH, de Graaf EJR, Punt CJA, et al. Long-term oncological and functional outcomes of chemoradiotherapy followed by organ-sparing transanal endoscopic microsurgery for distal rectal cancer: the CARTS study. JAMA Surg. 2019;154(1):47-54.

16. Barina A, De Paoli A, Delrio P, et al. Rectal sparing approach after preoperative radio- and/or chemotherapy (RESARCH) in patients with rectal cancer: a multicentre observational study. Tech Coloproctol. 2017;21(8):633-40.

17. Amin MB, Greene FL, Edge SB, et al. The eighth edition AJCC cancer staging manual: continuing to build a bridge from a population-based to a more "personalized" approach to cancer staging. CA A Cancer J Clin. 2017;67(2):93-9.

18. Mandard A-M, Dalibard F, Mandard J-C, et al. Pathologic assessment of tumor regression after preoperative chemoradiotherapy of esophageal carcinoma. Clinicopathologic correlations. Cancer. 1994;73(11):2680-6.
19. Lezoche E, Baldarelli M, Lezoche G, Paganini AM, Gesuita R, Guerrieri M. Randomized clinical trial of endoluminal locoregional resection versus laparoscopic total mesorectal excision for T2 rectal cancer after neoadjuvant therapy. $B r \quad J$ Surg. 2012;99(9):1211-8.

20. Smith JJ, Strombom P, Chow OS, et al. Assessment of a watchand-wait strategy for rectal cancer in patients with a complete response after neoadjuvant therapy. JAMA Oncol. 2019;5(4):e185896.

21. van der Valk MJM, Hilling DE, Bastiaannet E, et al. Long-term outcomes of clinical complete responders after neoadjuvant treatment for rectal cancer in the International Watch \& Wait Database (IWWD): an international multicentre registry study. Lancet. 2018;391(10139):2537-45.

22. Martens MH, Maas M, Heijnen LA, et al. Long-term outcome of an organ preservation program after neoadjuvant treatment for rectal cancer. J Natl Cancer Inst. 2016;108(12):djw171.

Publisher's Note Springer Nature remains neutral with regard to jurisdictional claims in published maps and institutional affiliations. 